\title{
Planetary populations according to the orbital angular momentum
}

\author{
João A. S. Amarante and Helio J. Rocha-Pinto \\ Universidade Federal do Rio de Janeiro, Observatório do Valongo \\ Lad. do Pedro Antônio 43, 20080-090 Rio de Janeiro RJ, Brazil \\ email: amarante@astro.ufrj.br, helio@astro.ufrj.br
}

\begin{abstract}
We investigate the angular momentum distribution of known exoplanetary systems, as a function of the planetary mass, orbital semimajor axis and metallicity of the host star. We find exoplanets seems to be classified according to at least two 'populations', with respect to their angular momentum properties. This classification is independent on the composition of the planet and seems to be valid for both jovian and neptunian planets, and probably can be extrapolated to the terrestrial planets of the Solar System. We analyse these 'populations' considering the phenomenon of planetary migration.
\end{abstract}

Keywords. exoplanets, planetary migration

\section{Introduction}

Presently, exoplanet sample sizes are sufficiently so large that what were previously seen as marginal trends can be checked against more detailed models of planetary formation. The first and apparently main trend discovered in these exoplanetary samples were the planet-metallicity connection (González 1997, Fischer \& Valenti 2005). The orbital period distribution also points to the existence of planetary 'populations' which may be linked to migration (Udry, Mayor \& Santos 2003). We show here that a new planetary classification emerge from the orbital angular momentum distibution.

\section{Angular Momentum Distribution}

Our sample was built with the planetary data available from a mid-2007 version of the online Catalog of Nearby Exoplanets Butler(2006). The sample is composed by 212 planets, from which 63 belong to one of the then known 26 exoplanetary systems. The orbital angular momentum $(\mathcal{L})$ was calculated from the published measurements for the mass, $M$, semimajor axis, $a$, and orbital period, $P$, for each planet.

An intriguing pattern can be seen in the mass-angular momentum log-log plot (Fig 1a): the planets seem to follow two, apparently parallel, linear relations. This implies that the distribution of $a^{2} / P$ values for the planetary sample used in this analysis should be approximately bimodal. Not unexpectedly, we have found two broad peaks in the $\log \left(a^{2} / P\right)$ distribution at 0.3 and $1.5 \mathrm{AU}^{2} \mathrm{yr}^{-1}$. Due to the fact that most of the known exoplanets moving in these 'intraterrestrial' orbits $\left(\frac{a^{2}}{P}<1 \mathrm{AU}^{2} \mathrm{yr}^{-1}\right)$ are jovians, we suspect that they correspond to a population of planets that have migrated from their formation radius into the inner planetary system.

Figure $1 \mathrm{~b}$ represents the p.d.f. for the mass bin marked on Figure 1a by dotted lines. We can clearly see a bimodal distribution. The solid lines in Fig 1a were estimated by a non-parametric regression method as follows. We have estimated the p.d.f. of the orbital angular momentum on several mass bins, using a Fourier kernel (Tarter \& Lock 1993). 

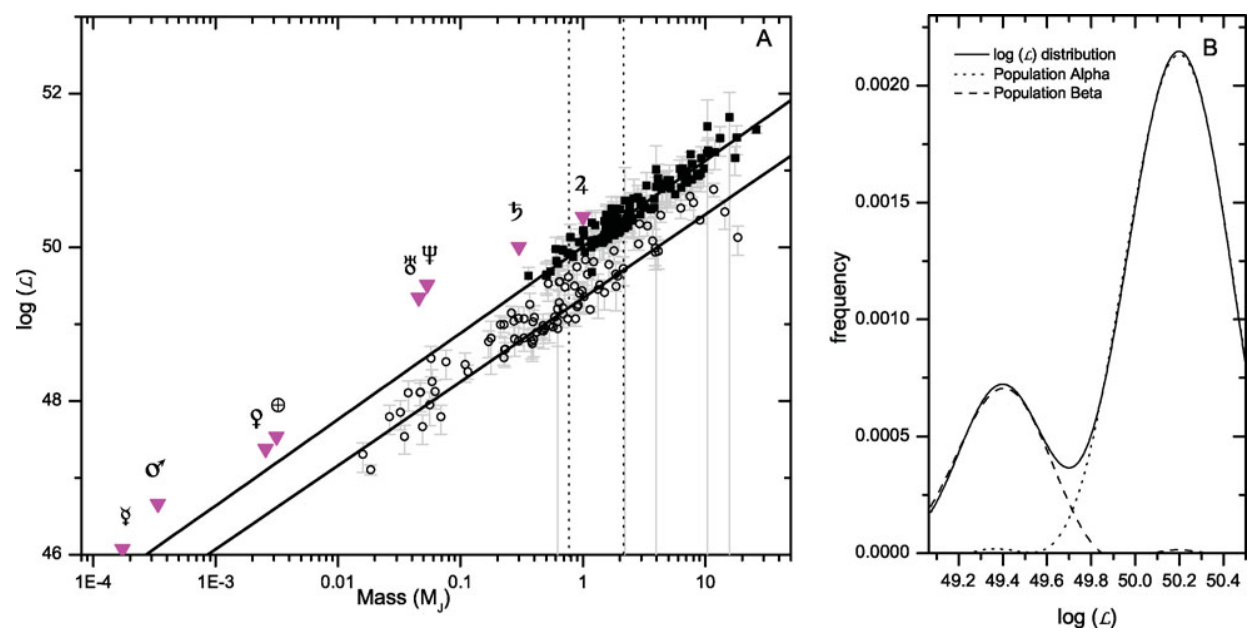

Figure 1. Panel A shows the mass-angular momentum diagram for planets known by mid-2007. Two apparent linear relations between mass and angular momentum can be seen (marked by solid lines). A nonparametric regression model was used to define these two relations. Solid squares and open circles planets represents planets having semimajor axis larger and smaller than 0.67 AU, respectively. Solar System planets are identified by their traditional symbols. Panel B shows the angular momentum p.d.f.s for the selected mass bin in Panel A as an example, showing the bimodality in $\mathcal{L}$.

For each mass bin we obtained the modes of the resulting $\mathcal{L}$ p.d.f.s. Then, we were able to define a relation between the orbital angular momentum and the planetary mass using a linear fit to these modes for each mass bin. From this, we can classify each planet in populations according to which $\mathcal{L}-M$ relation it probably follows. We will call these populations $\alpha$ and $\beta$. See an example of this separation in Fig $1 b$.

The distribution of planetary mass for both populations indicates that population $\beta$ has more low mass planets than population $\alpha$, suggesting that Population $\beta$ planets had a runaway migration process (for instance, see Trilling et al. 2002), explaning why Population $\beta$ presents less angular momentum than Population $\alpha$ which is mainly composed by more massive planets and would have had a different migration process. The Kolmogorov-Smirnov test on the cumulative distribution of planetary host stars mass indicate that we can reject the hyphotesis that the distributions come from the same population with a $95 \%$ confidence level. This can also indicate that both populations may have been through different kinds of migration (e.g., Kennedy \& Kenyon 2009).

\section{References}

Butler R. P., et al. 2006, ApJ, 646, 505

González, G. 1997, MNRAS, 285, 403

Fisher, D. A. \& Valenti, J. 2005, ApJ, 622, 1102

Kennedy, G. M. \& Kenyon, S. J., ApJ, 695, 1210

Tarter, M. E. \& Lock, M. D., 1993, Model-Free Curve Estimation, Chapman \& Hall: New York, p. 55

Trilling, D., Lunine, J., \& Benz, W. 2002,

Udry, S., Mayor, M., \& Santos, N. C. 2003 